\title{
The study of the relationship between life limiting factor and stress level for FGH96
}

\author{
Didi. Yang ${ }^{1}$, Yi. Shi ${ }^{1}$, Guolei. Miao ${ }^{2}$, Xiaoguang. Yang ${ }^{1}$ and Duoqi.Shi ${ }^{1}$ \\ ${ }^{1}$ Beihang University - Beijing (China) \\ ${ }^{2}$ Chengdu Holy Industry \& Commerce Corp. Ltd. (Group) - Chengdu (China)
}

\begin{abstract}
FGH96 is a Chinese made powder metallurgy nickel based superalloy. This reserach aims to investigate the effect of stress on the microstructure of the worst fatigue life at $600^{\circ} \mathrm{C}$. The specimens were first polished by abrasive paper and then electropolished to elimate the effect of surface residual stress. The fatigue tests were conducted at $600^{\circ} \mathrm{C}$, and the loading ratio was 0.05 at the frequency of $5 \mathrm{~Hz}$. Tests were conducted at $900,1000,1100,1200 \mathrm{MPa}$ respectively. The fatigue life under the same condition were compared and the fractography of specimens were examined under SEM to further identify the lifelimiting factors of the material. The initiation mode transferred from facet initiated to inclusion initiated from lower stress level to higher stress level. Meanwhile, the initiation position transferred from internal to surface with the increase of stress level. This means at higher stress level, fatigue life is mainly limited by inclusion at surface while at lower stress level the limit is controlled by internal facet.
\end{abstract}

\section{Introduction}

FGH96 is one of the active-disc materials. Many stu-dies have been conducted on the fatigue life of FGH96 materials. Large to full-size and sub-dimensions ${ }^{[1]}$, down to sample level ${ }^{[2]}$, there are many researches on factors affecting fatigue life. In order to deeply study the microstructural factors that affect fatigue life, this paper conducts SEM studies on fatigue fractures, by comparing the impact factors of the worst fatigue life under the same load, and the difference in the worst fatigue life factors under different loads.

Fatigue life is determined by crack initiation life and crack propagation life. The aim of this study is to explore the effect of fatigue crack initiation modes and position and the crack propagation path on fatigue life. The main modes of crack initiation investigated this time are facet and inclusion initiation. Under the normal stress conditions, facet initiation may occur. In the manufacturing process, nonmetallic and heterogenous metal inclusions will be introduced in FGH96. Stress concentration caused by inclusions make cracks appear. Crack initiation positions can be divided into surface, subsurface, and internal. The crack propagation path is mainly investi-gated by macroscopic fractures. The crack propagation path is influenced by environmental factors and stress factors.

\section{Testing material and experiment procedure}

\section{1 materials}

The material tested is a high-temperature nickel-base superalloy named FGH96. FGH96 is China's secondgeneration polycrystalline powder metallurgy superalloy, mainly used for aero-engine turbine discs. The chemical compositions is given in Table 1 FGH96 was manufactured by vacuum smelting alloy, atomizing alloy followed by isostatically pressing, extruding and isothermally forging ${ }^{[3]}$.

EBSD measurements were used to map the grain size and characterize the grain orientation. The obtained microstructure is shown in Fig. 1. Macroscopically, the material is isotropic and contains twins. The average grain size is $23 \mathrm{~mm}$. There is no defect information such as in-clusions and pores been observed, for the limited scanning area of EBSD. Detailed information has been introduced in previous work ${ }^{[4]}$.

Table 1. Chemical composition of experimental steels ( $\mathrm{wt} \%$ ).

\begin{tabular}{c|c|c|c}
\hline $\mathrm{Cr}$ & $\mathrm{Co}$ & $\mathrm{W}$ & $\mathrm{Mo}$ \\
\hline $15.5 \sim 16.5$ & $12.5 \sim 13.5$ & $3.8 \sim 4.2$ & $3.8 \sim 4.2$ \\
\hline $\mathrm{Al}$ & $\mathrm{Nb}$ & $\mathrm{Ti}$ & $\mathrm{Ni}$ \\
\hline $2.0 \sim 2.4$ & $0.6 \sim 1.0$ & $3.5 \sim 3.9$ & Bal. \\
\hline
\end{tabular}




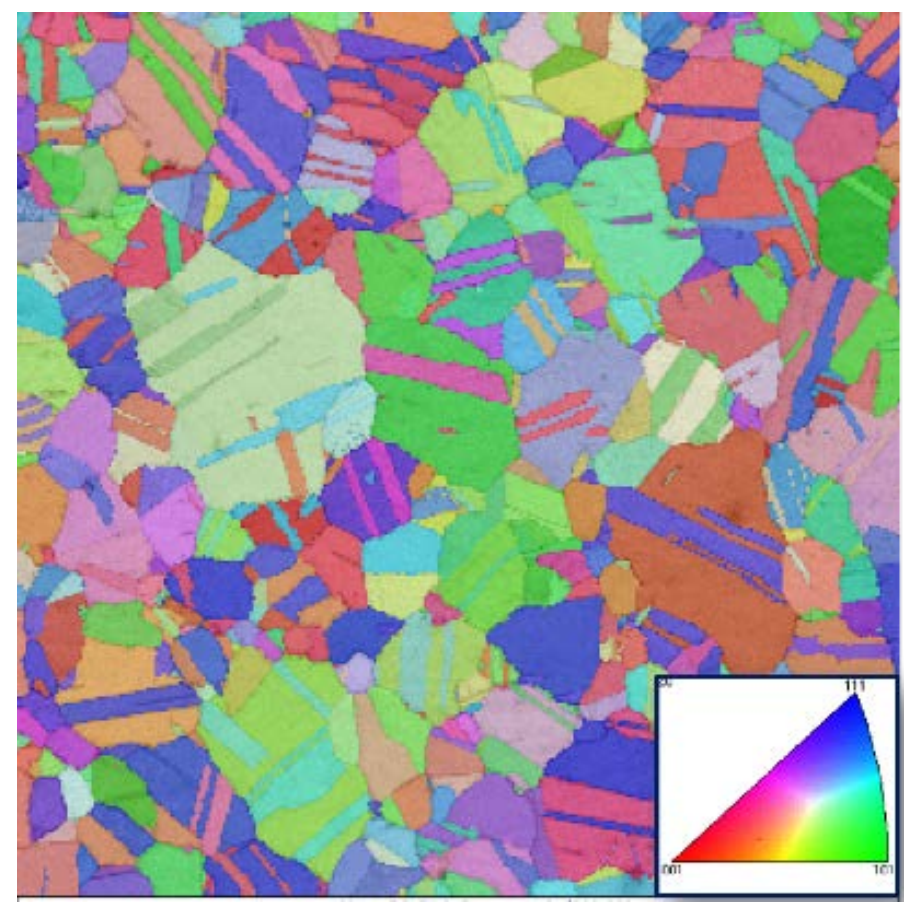

Fig. 1. Microstructure of the FGH96 (grain orientation and size)

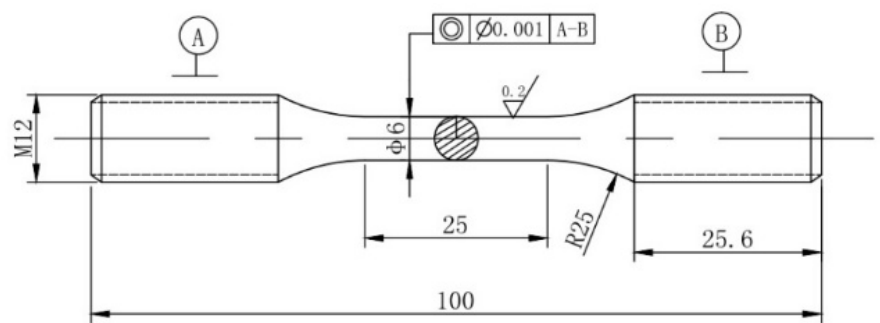

Fig. 2. The shape and dimensions of the specimen

\section{2 experiment procedure}

All fatigue specimens were machined from the same disk. The geometry of the specimen is shown in Fig. 2. To eliminate the impact of processing, e.g. surface residual stress, surface scratches and roughness, specimens were roughly polished using increasingly fine abrasive paper up to 2000 grit followed by electropolishing. Electro-polishing was conducted using an electrolyte of $55 \%$ ethanol with $35 \%$ butyl cellusolve and $10 \%$ perchloric acid at $25 \mathrm{~V}$ and $20{ }^{\circ} \mathrm{C}$. The measurement results show that the surface of the specimen participates in stress below -100 MPa, and the effect of residual stress can be ignored.

The fatigue tests were conducted at $600{ }^{\circ} \mathrm{C}$ using a SHIMADZU servo-hydraulic test system according to the test standard. The tests were performed in stress control at the frequency of $5 \mathrm{~Hz}$, the ratio (R) of 0.05 . Nowadays the maximum stress of the aero-engine turbine disk is approximately $1100 \mathrm{Mpa}^{[5]}$ (with the rotation speed $10000 \mathrm{r} / \mathrm{min}$ ). And considering the existing test data ${ }^{[6,7]}$, the stress levels were set at $1100 \mathrm{MPa}$ and $1200 \mathrm{MPa}$ for the low cycle fatigue and 900MPa and $1000 \mathrm{MPa}$ for the high cycle fatigue respectively.

\section{Experiment results}

\section{1 stress and life}

With the increase of the stress, the corresponding life reduced rapidly. And the variability of the fatigue life showed a trend of decreasing first and then increasing. The reason was given in previous work ${ }^{[4]}$.

\section{2 fatigue fracture analysis}

To study the factors that limit the fatigue life, the fatigue fracture surfaces of all the tests were investigated by SEM analysis, as shown in Fig. 3, Fig. 4, Fig. 5 and Fig. 6. The main object of the study is the stage of crack initiation. At the same time, the state of the crack propagation stage under different stresses has also been studied.

Through the analysis of the fracture map, there are two initiation modes: (a) facet initiated; (b) inclusion initiated, and three initiation positions: (a) internal initiated (b) sub-surface initiated and surface initiated. With the increase of the stress level, the crack initiation modes of the worst-life changes from facet initiation to

\footnotetext{
*Corresponding author: yangdidi4521@163.com
} 
inclusion initiation. In addition, the crack initiation position starts from the internal to the surface. Table 2 shows the initiation modes of the worst life test specimen at each stress level.

At lower stress level, the facet initiation position mostly appear in the internal. While, at the higher stress level, they mostly appear on the surface, consistent with the reaction of inclusions to stress. It has been mentioned in previous work that when the crack initiation position approaches the surface, the crack growth will be influenced by environmental factors (oxygen, etc.), so the life is shorter than the internal crack. But at $900 \mathrm{MPa}$, there was a completely opposite result. As the crack approached the surface, the fatigue life increased (as shown in Fig. 8) and the lifetime of the crack near the subsurface was 18 times that of the internal.

In addition, at higher stress level, the shape of the crack no longer maintains an arc-shaped outward extension at the later stage of the extension. The shorter the fatigue lives are, the narrower the crack surface is, showing a "bamboo shoot" shape, as shown in Fig. 7. The "thinner" the bamboo shoots are, the shorter the fatigue life is.

\section{Discussion}

The relationship between stress and life is plotted in semi-logarithmic coordinates, as shown in Fig. 9 and Fig. 10. The S-N curve obtained by exponential calculation is a straight line. Using the 3-sigma theory, the worst life curve with a confidence of $99.87 \%$ can be calculated. To study the factors limiting the fatigue life, focusing on the part between the S-N curve and the worst life curve. The microscopic factors that limit fatigue life will be explored separately from the crack initiation modes and the initi-ation position.

\subsection{Initiation modes}

As shown in the Fig. 9, the initiation modes transfer from facets to inclusion when the stress increasing. At $900 \mathrm{MPa}$ and $1000 \mathrm{MPa}$, the initiation modes of cracks are facet, as shown in Fig. 3 and Fig. 4. And at $1100 \mathrm{MPa}$ and $1200 \mathrm{MPa}$, the initiation modes of cracks are inclusion, as shown in Fig. 5 and Fig. 6. Crack initiation mechanism of the inclusion initiation and facet initiation are very different. The facet crack initiation is that under the normal stress, the crystal plane with the weakest atomic bonding cracks. Meanwhile, there are usually three

cond-

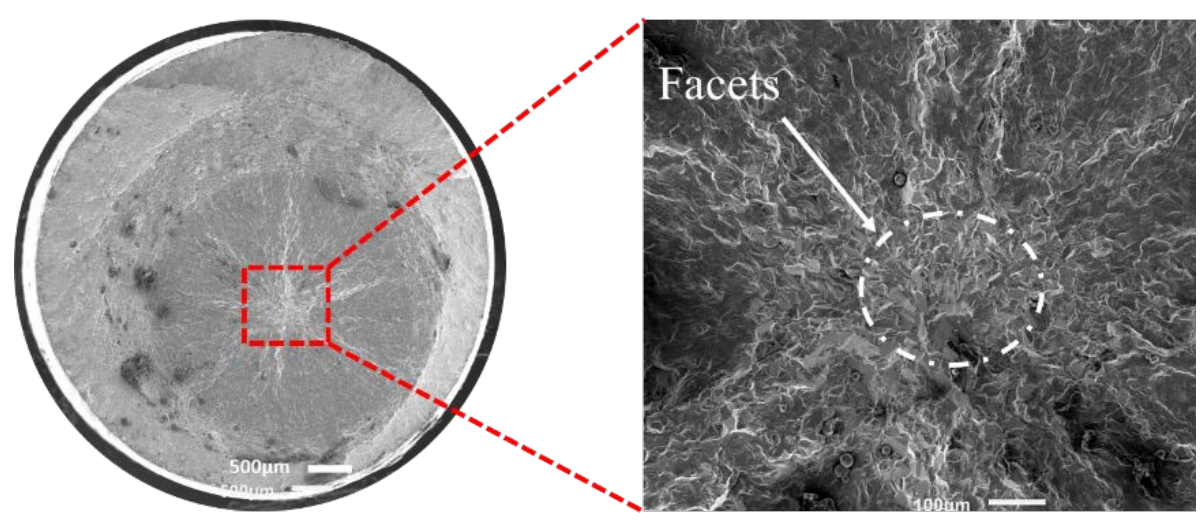

Fig. 3. SEM image of FGH96 fatigue fracture at $900 \mathrm{Mpa}$ (left: low magnification. right: high magnification)

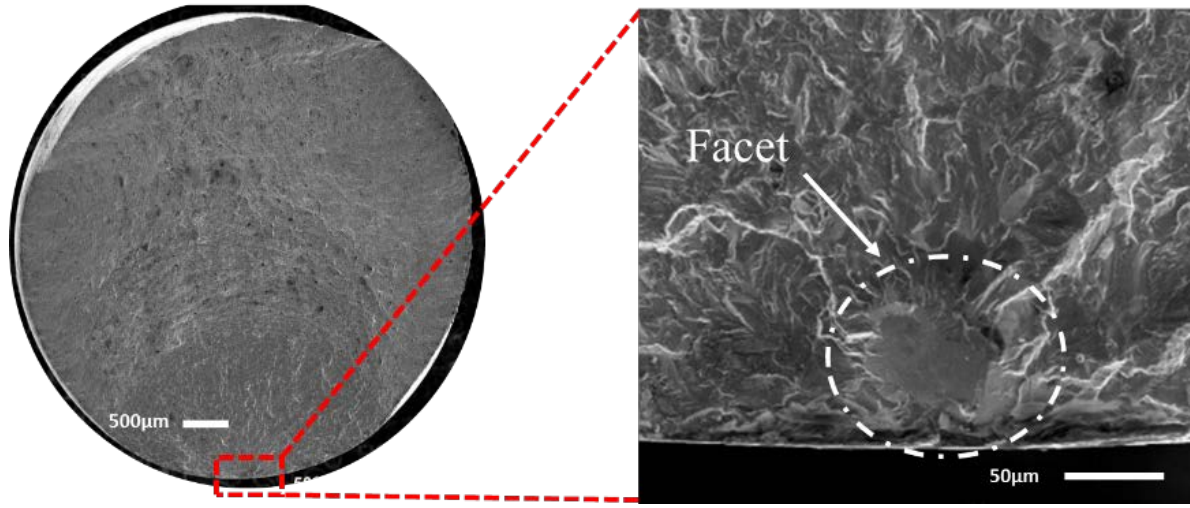

Fig. 4. SEM image of FGH96 fatigue fracture at $1000 \mathrm{Mpa}$ (left: low magnification. right: high magnification) 


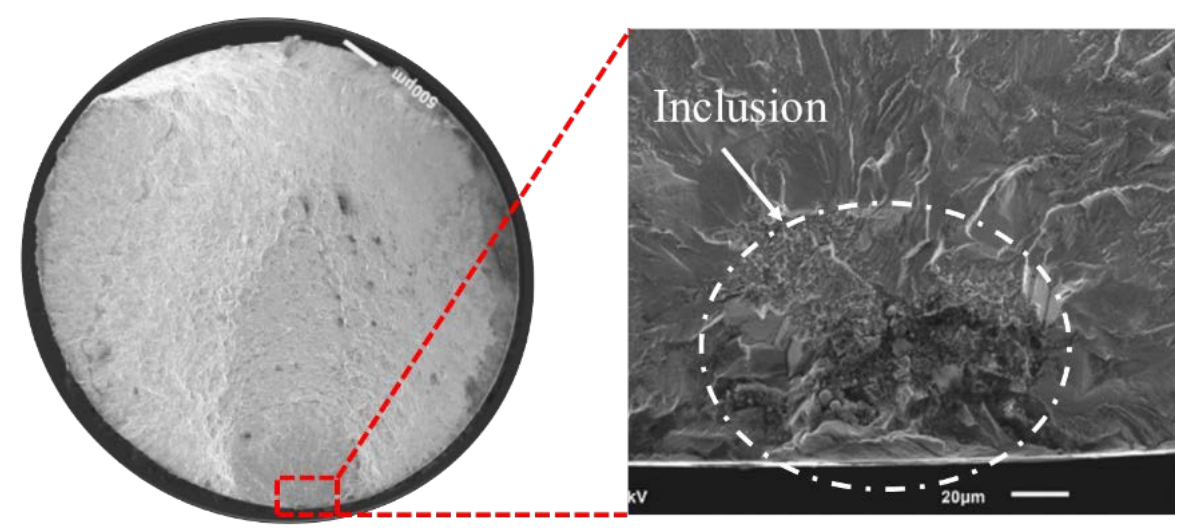

Fig. 5. SEM image of FGH96 fatigue fracture at $1100 \mathrm{Mpa}$ (left: low magnification. right: high magnification)

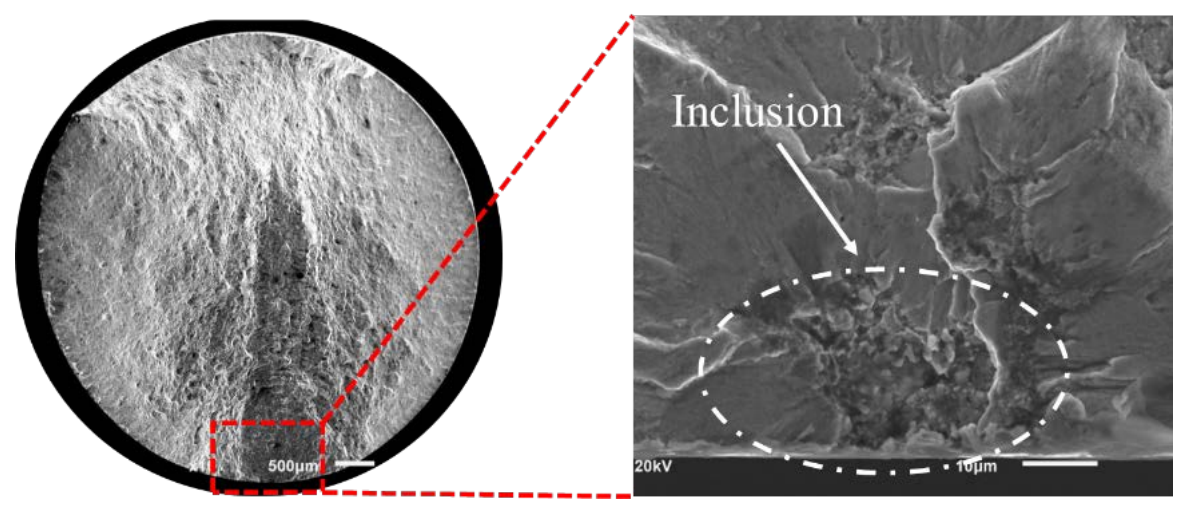

Fig. 6. SEM image of FGH96 fatigue fracture at 1200 Mpa (left: low magnification. right: high magnification)
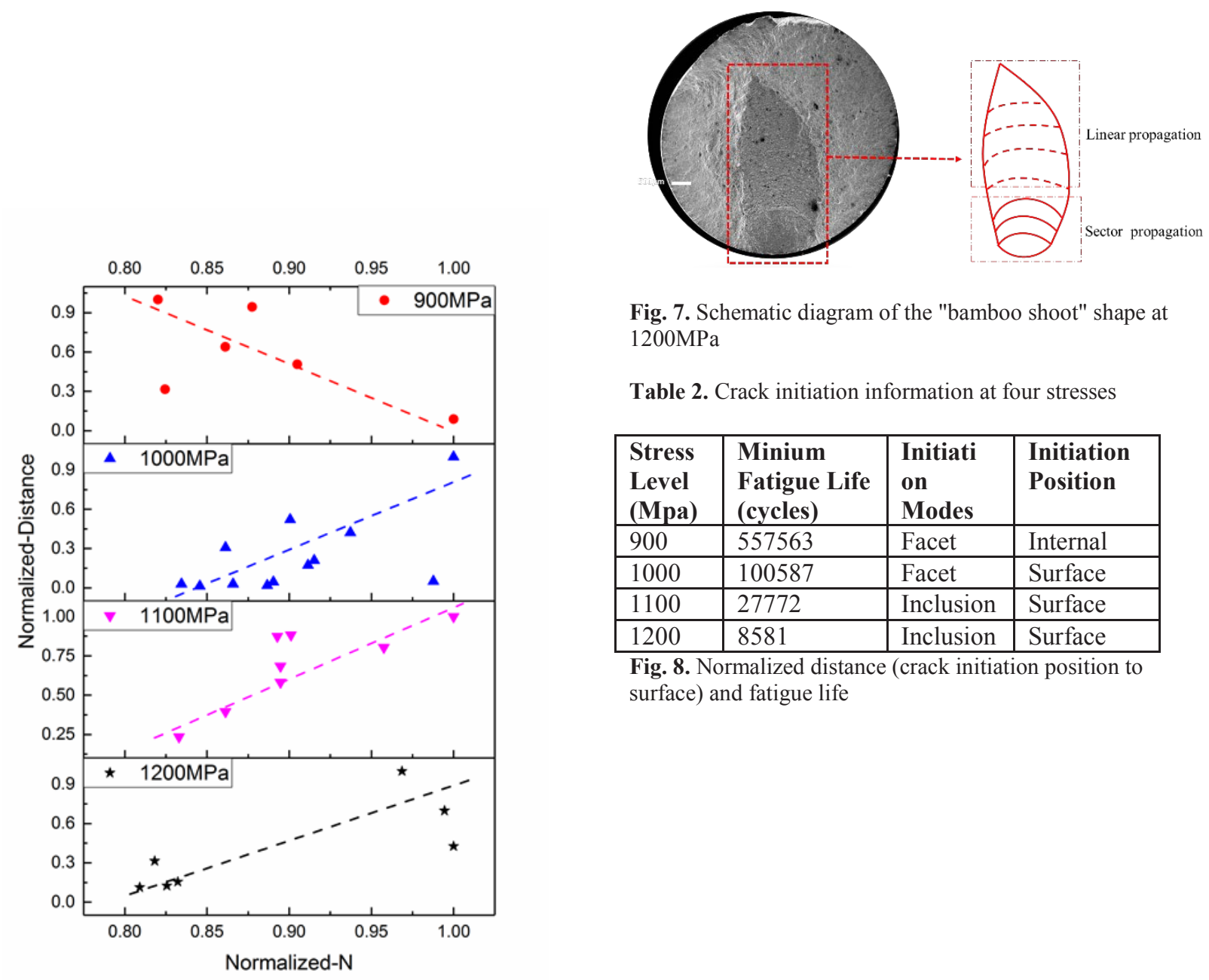

Fig. 7. Schematic diagram of the "bamboo shoot" shape at $1200 \mathrm{MPa}$

Table 2. Crack initiation information at four stresses

\begin{tabular}{|l|l|l|l|}
\hline $\begin{array}{l}\text { Stress } \\
\text { Level } \\
\text { (Mpa) }\end{array}$ & $\begin{array}{l}\text { Minium } \\
\text { Fatigue Life } \\
\text { (cycles) }\end{array}$ & $\begin{array}{l}\text { Initiati } \\
\text { on } \\
\text { Modes }\end{array}$ & $\begin{array}{l}\text { Initiation } \\
\text { Position }\end{array}$ \\
\hline 900 & 557563 & Facet & Internal \\
\hline 1000 & 100587 & Facet & Surface \\
\hline 1100 & 27772 & Inclusion & Surface \\
\hline 1200 & 8581 & Inclusion & Surface \\
\hline
\end{tabular}

Fig. 8. Normalized distance (crack initiation position to surface) and fatigue life 


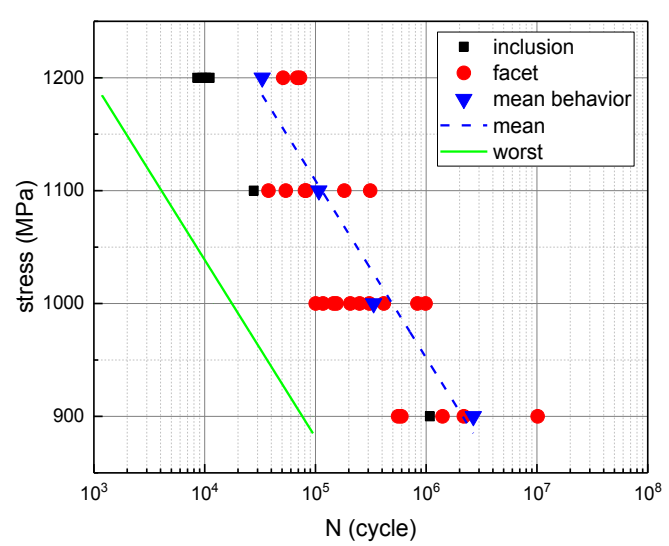

Fig. 9. Comparing the initiation modes at four stresses

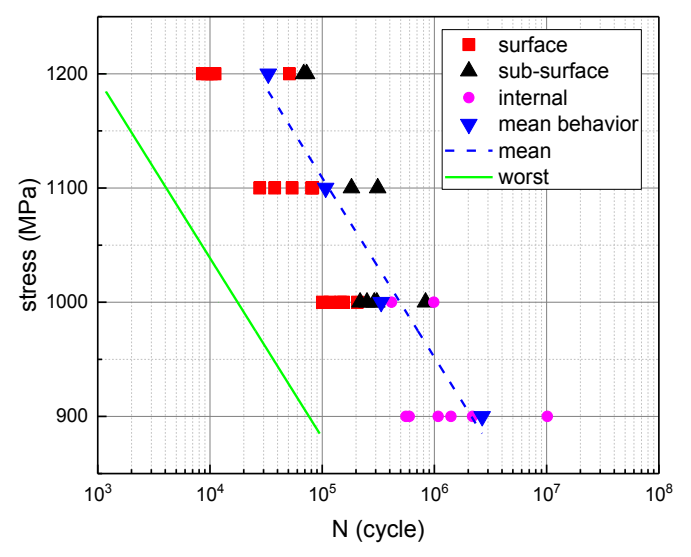

Fig. 10. Comparing the initiation modes at four stresses

itions that Nickel-based powder superalloy initiating cracks at inclusions: (a) cracking of the inclusion itself, (b) cracking of the inclusion/substrate interface, (c) cracking of the substrate adjacent to the inclusion ${ }^{[8]}$.

Forming a crack surface requires absorbing enough energy, according to Griffith theory. Energy absorption is related to load and time. Stress concentration occurs around the inclusions. But, when the external load is small, the stress concentration caused by the inclusion does not have a great influence. Meanwhile, the energy required to break the atomic bonding force forming a facet is smaller than the energy needed to initiate crack near an inclusion. So the facet crack initiation occurs first at lower stress level. As the load increases, the stress around the inclu-sion caused by stress concentration increases rapidly, and the difference with the average stress increased. Then the material close to the inclusion first obtains enough energy to initiate cracks. This can explain the initiation of cracks around inclusions at higher stress level.

\subsection{Initiation position}

The initiation position transfers from internal to surface when the stress increases. At higher stress levels, the crack initiation positions are mainly surface and subsurface, as shown in Fig. 4, Fig. 5, Fig. 6. As analyzed in 4.1 section, with the increase of stress, inclusions become the main micro-structural factor that limits the fatigue life. And the stress concentration factor caused by inclusions becomes more evident with the inclusion approaching the surface ${ }^{[9]}$, thus the surface and subsurface of the material are more prone to cracks. When the stress level is not too high ( at $900 \mathrm{MPa}$ ), the area (volume) of the inclusion can offset the influence of the stress concentration factor caused by the position. Therefore, the position of the crack initiation is random and may even appear inside, as shown in Fig. 3.

When at lower stress level is, such as $900 \mathrm{MPa}$, the main mode of initiation is facet. The facet occurs mainly at large grains and grain boundaries, and these features are common within the specimen. So cracks usually initiated in the internal of the material.

\section{3 crack growth}

The SEM result of the worst life specimen in $1200 \mathrm{MPa}$ was shown in Fig. 11.The narrower the crack surface is, the faster the critical length can be reached at the same energy input, making the remaining distance of the specimen (i.e. the length of the line in the rectangular frame in Fig.11) less than the safety distance. Then the break occurred. On the other hand, sharp crack surfaces increase stress concentration, making crack growth faster and fatigue life worse.

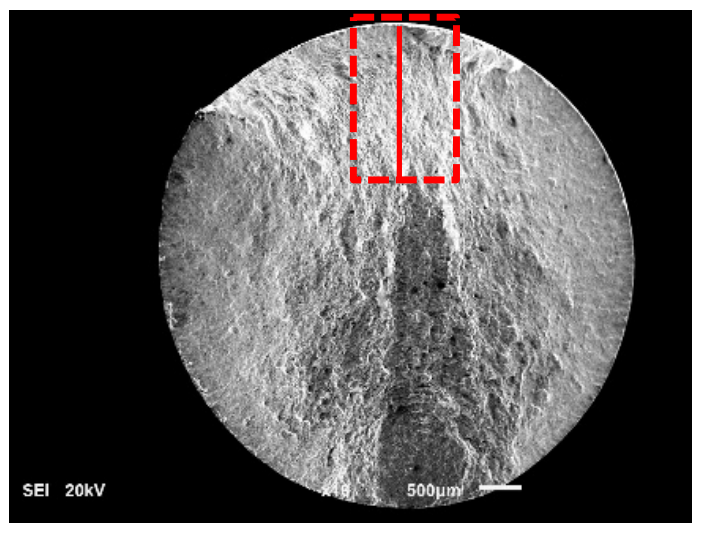

Fig. 11. Fracture diagram of worst life at $1200 \mathrm{MPa}$

\section{Conclusion}

By comparing FGH96 fatigue life and fracture microstructure, the conclusions are as follows:

1 ) The main limiting factors of fatigue life are inclusion defects and crack initiation caused by facets. At higher stress levels, inclusions are the main microscopic factors limiting fatigue life; at lower stress levels, facet is the microscopic factor limiting fatigue life;

2 ) At higher stress levels, fatigue cracks are mostly initiated on the surface, while at lower stress levels, cracks are initiated more internally. 
3 ) The main factor limiting the fatigue life is the relation-ship between inclusions and stress. At lower stress levels, inclusions do not substantially affect the worst life, and the inherent properties of the material (atomic bond weakest resultant force) cause crack initiation, limit-ing fatigue life. At higher stress levels, inclusion limits fatigue life.

4 ) At the higher stress level, the closer the initiation position is to the surface, the shorter the life is; while at the lower stress level, the closer the initiation position is to the surface, the longer the lifetime is.

The authors would like to thank the sponsorship by National Program on Key Basic Research Project (973 Program) (No. 2015CB057401).

\section{References}

1. Y. Feng, C. Wu, W. Gao, W. Chen, J. Aerosp. Power. 27:628-634(2012)

2. D. Wei, X. Yang, Y. Wang, et al, J. Aerosp. Power, 22: 425-430(2007)

3. China Aviation Material Handbook Committee. China Aviation Material Handbook. 5, 29 (2002)

4. G. Miao, X. Yang, D. Shi, Mater. Sci. Eng., A, 668:6672(2016)

5. T. Liu, Q. Deng, Y. Liu, J. Mech. Res. Appl, 28:94-96. (2015)

6. Cashman G T, Int. J. Fatigue, 32:492-496 (2010)

7. S.K. Jha, M.J. Caton, J.M. Larsen. AFRL-RX-WP-TM2010-4084, 57-72 (2008)

8. Y. Zeng, M. Zhang, J. Dong, et al, J. Mater. Eng,353358:1185-1190 (2005)

9. S. Yuan, Beijing: BUAA (2014) 